\title{
Comorbidade entre Uso de Álcool e Outras Drogas, Transtornos Psiquiátricos e Comportamento Suicida: Uma Revisão
}

\section{Comorbidity between the Use of Alcohol and Other Substances, Psychiatric Disorders and Suicide Behavior: A Review}

\section{Comorbilidad entre el Uso de Alcohol y Otras Drogas, Trastornos Psiquiátricos y Comportamiento Suicida: Una Revisión}

\author{
Juliana Gomes da Cunha Baltar \\ Alexandra Iglesias \\ Elizeu Batista Borloti \\ Universidade Federal do Espírito Santo
}

\begin{abstract}
Resumo
Estudos e dados governamentais apontam possível associação entre uso abusivo de álcool e outras substâncias e comportamento suicida, com consequente impacto em diversos âmbitos, como o econômico e o da saúde pública. Diante desse panorama, objetivou-se descrever a comorbidade a partir de uma revisão de literatura, de modo a apontar aspectos relevantes para sua prevenção. Para tanto, realizou-se busca avançada no Portal de Periódicos CAPES, a partir da qual 17 artigos foram selecionados e agrupados em cinco temas. Em suma, compareceu nos estudos revisados o cuidado em assinalar subgrupos de risco para o suicídio entre os usuários de álcool e outras drogas, que se constituem como público-alvo para intervenções preventivas de agravos e de promoção à saúde. Palavras-chave: suicídio, álcool etílico, drogas, prevenção
\end{abstract}

\begin{abstract}
Studies and government data point out a possible association between the abuse of alcohol and other substances and suicidal behavior, with a consequent impact on various fields, such as the economic field and public health. Facing this situation, this article aims to describe this comorbidity the way it is described in the current bibliography, in order to point out relevant aspects for its prevention. For this purpose, an advanced search was conducted on the CAPES's scientific paper directory from which 17 articles were selected and grouped into five themes. In short, the reviewed studies demonstrated the caution of marking suicide risk subgroups among psychoactive substance users, which constitute a target group for preventive interventions of aggravations and promotion of health.
\end{abstract}

Keywords: suicide, ethyl alcohol, drugs, prevention

\section{Resumen}

Estudios y datos gubernamentales señalan posible asociación entre el uso abusivo de alcohol y otras sustancias y comportamiento suicida, con consecuente impacto en diversos ámbitos, como el económico y el de la salud pública. Ante este panorama, se objetivó describir la comorbilidad a partir de una revisión de literatura, para apuntar aspectos relevantes para su prevención. Para ello, se realizó una búsqueda avanzada en el Portal de Periódicos CAPES, a partir de la cual 17 artículos fueron seleccionados y agrupados en cinco temas. En resumen, se observó en los estudios revisados el cuidado en señalar subgrupos de riesgo para el suicidio entre los usuarios de alcohol y otras drogas, que se constituyen como público objetivo para intervenciones preventivas de agravios y de promoción a la salud.

Palabras clave: suicidio, alcohol etílico, drogas, prevención

\footnotetext{
${ }^{1}$ Endereço de contato: Rua José Luiz Gabeira, 170, Bairro Barro Vermelho, Vitória, ES, CEP 29057-570. E-mail: juliana88gomes@gmail.com
} 


\section{Introdução}

É possível considerar a dependência e o abuso de drogas como um problema de saúde pública relevante. Apesar disso, não é um fenômeno novo, já que o uso de álcool e outras drogas e, por conseguinte, a dependência dessas substâncias² acompanham o homem desde os primórdios, sendo relatados por todas as culturas conhecidas (Bastos, Bertoni, Hacker, \& Grupo de Estudos em População, Sexualidade e Aids, 2008; Déa, Santos, Itakura, \& Olic, 2004; Guimarães, Godinho, Cruz, Kappann, \& Tosta, 2004).

Em relação ao Brasil, Déa et al. (2004) apontam que o padrão de consumo de drogas ainda não é comparável ao de países desenvolvidos, apesar do grande crescimento recente. Bessa, Boarati e Scivoletto (2011) ressaltam diferenças sociais, econômicas e culturais sobre esse consumo entre brasileiros e pessoas de outras nacionalidades. Em relação ao Brasil e aos Estados Unidos, verificaram que o uso de todas as substâncias - exceto inalantes - é maior neste último do que no primeiro, o que possivelmente se relaciona a uma maior oferta (disponibilidade) e um maior poder aquisitivo para adquiri-las (acessibilidade).

Hipoteticamente, pode-se relacionar o consumo expressivo de inalantes no Brasil ao contexto social permeado por desigualdades. Nesse sentido, pessoas em situação de rua, por exemplo, diante da falta de investimento do governo em políticas de garantia dos direitos sociais básicos, usam essas substâncias em um "processo de anestesia" (Escorel, 1999, p. 12) para permanência e sobrevivência nas ruas.

O abuso de drogas e a dependência química podem acarretar severas consequências sociais e individuais, como o desenvolvimento de transtornos psiquiátricos e comportamentos de risco (Hess, Almeida, \& Moraes, 2012). Nesse contexto, Hess et al. (2012) destacam os transtornos de ansiedade e depressivos e a alta prevalência de risco de suicídio.

Ao endossar o alto risco de ocorrência de suicídio entre pessoas com dependência química, Saide (2011) associa tal situação a dificuldades na regulação emocional e do humor, bem como à comorbidade com o transtorno depressivo maior. Assim, em um quadro de coexistência entre estado depressivo e dependência química, é possível notar que se configura uma realidade circular, em que um reforça e realimenta o outro, quando não tratados cuidadosamente. De acordo com o exposto, faz-se necessária a atenção a diversos contextos sociais, tendo em vista a forte associação entre transtornos relacionados ao uso de substância e o comportamento suicida. De modo mais específico, é dado pela Organização Mundial de Saúde (OMS, 2000, n. p.) que:

em média, um único suicídio afeta pelo menos outras seis pessoas. Se um suicídio ocorre em uma escola ou em algum local de trabalho, tem impacto em centenas de pessoas. ... O custo do suicídio pode ser estimado em termos de DALYs (disability-adjusted-life-years, ou anos de vida ajustados às limitações). De acordo com esse indicador, em 1998 o suicídio foi responsável por 1,8\% do custo total de doenças em todo o mundo, variando entre 2,3\% em países de alta renda a 1,7\% em países de baixa renda. Esse é um

\footnotetext{
${ }^{2}$ Cabe diferenciar uso, abuso e dependência de álcool e outras drogas ou dependência química. Compreendese uso como uma experiência esporádica. Quando se torna frequente, chegando a trazer algum tipo de prejuízo ao indivíduo, o uso passa a ser considerado abuso. Por fim, a dependência química é configurada quando o indivíduo passa a ter severas dificuldades para se manter sem o consumo da substância (Brasil, 2017).
} 
custo equivalente ao das guerras e homicídios, grosseiramente aproxima-se do dobro do custo do diabetes, e equivale ao custo do trauma e asfixia neonatais.

Em adição, Silva et al. (2010) apontam a necessidade de priorizar o suicídio como problemática de saúde pública, já que está mundialmente entre as dez primeiras causas de morte em todas as idades e entre as três primeiras na faixa entre 15 e 35 anos. Diante desses dados, os autores reafirmam o uso abusivo de álcool e outras drogas como predisponente ao suicídio.

Ademais, Swendsen et al. (1998), Boschloo et al. (2011), Kotov, Gamez, Schmidt e Watson (2010) e Vicente, Nunes, Viñas, Freitas e Saraiva (2001) se dedicaram a estudar, em tempos e de modos diversos, a relação específica entre abuso/dependência de álcool e transtornos psiquiátricos como ansiedade e depressão. Como resultado, Swendsen et al. (1998) observaram que a presença de transtornos de ansiedade ou depressão é consistentemente associada ao aumento moderado dos sintomas de abuso ou dependência de álcool.

Boschloo et al. (2011), Kotov et al. (2010) e Vicente et al. (2001), por sua vez, assinalam diferenças significativas nas relações entre transtornos psiquiátricos e padrões de consumo de álcool, especificamente abuso e dependência. É possível, entretanto, encontrar dissenso de causa-efeito nas afirmações de Boschloo et al. (2011) e Kotov et al. (2010) sobre a relação entre dependência química e transtorno psiquiátrico: os primeiros afirmaram na época que, em suma, a dependência de álcool, mas não o abuso, é mais comum entre as pessoas ansiosas e/ou deprimidas do que entre as que não o são; enquanto os segundos ressaltaram que a dependência é fator de risco para transtornos de ansiedade e/ou depressão, enquanto o abuso não o é.

Diante dessa controvérsia que permeia os elementos causador e de causa na relação entre transtornos psiquiátricos e dependência de álcool - que estendemos aqui para outras drogas -, cabe mencionar Hess et al. (2012, pp. 174-175), que fazem as seguintes afirmações acerca dos sintomas decorrentes dos mencionados transtornos:

Na literatura psiquiátrica, observam-se duas hipóteses sobre a frequência desses sintomas entre usuários de drogas. Alguns autores sugerem que a existência prévia dessa sintomatologia pode estar associada ao aumento das chances de consumo de drogas como forma de redução do sofrimento emocional decorrente dessas doenças mentais, enquanto que outros, por sua vez, destacam uma relação direta do aumento dos transtornos internalizantes como consequência do efeito das substâncias psicoativas no sistema nervoso central.

Por fim, os achados de Vicente et al. (2001), em estudo comparativo entre pessoas em abuso e dependência de álcool, concordam com os de Kotov et al. (2010), pois apontam que os dependentes são propensos a maior gravidade de depressão e ansiedade, além de apresentarem maiores índices de desesperança e ideação suicida. De todo modo, a OMS (2000) aponta que tanto o abuso quanto a dependência do álcool são diagnósticos frequentes entre quem cometeu suicídio, especialmente entre jovens. Ainda segundo a OMS, existem explicações psicológicas, sociais e biológicas para a correlação entre alcoolismo e comportamento suicida, sendo relacionados ao agravamento dos riscos certos fatores específicos, como: alto nível de dependência, humor depressivo, história crônica de alcoolismo e início precoce de uso do álcool. 
Tendo em vista esse panorama que aponta correlação entre o uso abusivo ou a dependência de álcool e outras drogas e o comportamento suicida, objetivou-se neste estudo descrever tal fator a partir de uma revisão do que tem sido produzido e indicado pela literatura científica acerca da comorbidade apresentada, de modo a apontar aspectos relevantes para sua prevenção e para a promoção à saúde dessas pessoas, como indicam essas leituras analisadas ${ }^{3}$.

\section{Método}

O presente estudo trata-se de revisão bibliográfica a respeito da correlação entre as temáticas uso, abuso ou dependência de álcool e outras drogas e transtornos psiquiátricos, como o comportamento suicida. O artigo foi produzido durante disciplina do doutorado da primeira autora, sob orientação dos demais, enquanto eram trabalhados aspectos da saúde mental. A dependência química e o suicídio se inserem nas práticas profissionais e nos interesses acadêmicos dos três autores. A primeira autora tinha por objetivo, à época da disciplina, pesquisar efeitos de uma intervenção psicológica no cenário de um CAPS ad sobre sintomas de depressão, ansiedade e ideação suicida de sujeitos em tratamento nesse serviço, o que veio a suscitar o interesse na dita associação.

Para tanto, realizou-se busca avançada no Portal de Periódicos Capes, utilizando os descritores substance abuse no campo título e suicide no campo assunto, restringindo a busca a artigos publicados entre os anos de 2006 e 2016. Esses descritores foram combinados pelo operador booleano and. Ao todo, foram encontradas 50 publicações que abordavam a temática em questão. Em adição, buscaram-se os mesmos descritores em português, a saber: abuso de substância no título e suicídio no campo assunto. Quando pesquisados termos em português, todavia, não houve retorno de artigos revisados por pares. A escassez foi expressiva, o que levou a restringir a busca aos termos em inglês, e, consequentemente, fez com que não aparecessem artigos locais.

Seguindo na seleção dos 50 artigos, foi feita checagem inicial dos resumos, a fim de detectar os trabalhos que tratassem diretamente da relação entre abuso ou dependência de substâncias e suicídio. Foram incluídos artigos publicados na íntegra em periódicos científicos indexados no portal de periódicos Capes, a partir de 2006, ano de publicação do "Prevenção do suicídio: manual dirigido a profissionais das equipes de saúde mental" pelo Ministério da Saúde (Brasil, 2006). Foram excluídos trabalhos de conclusão de curso, dissertações de mestrado, teses de doutorado, capítulos de livro, bem como artigos que tratavam dos dois fenômenos relacionados a outros, como o artigo de Reed, Nugent e Cooper (2015), em que abuso de substâncias e tentativa de suicídio comparecem como repercussões de bullying e cyberbullying entre adolescentes norte-americanos ${ }^{4}$.

A partir desses critérios, foi possível chegar a 17 artigos revisados, que serão apresentados agrupadamente em temas, de acordo com as similaridades de seus conteúdos, como indicado por Rother (2007).

\footnotetext{
${ }^{3}$ O estudo foi possível por meio de bolsa de doutorado da primeira autora, concedida pela Fundação de Amparo à Pesquisa e Inovação do Espírito Santo (FAPES).

${ }^{4}$ As nacionalidades dos artigos revisados foram indicadas ao longo deste texto para fins de contextualização da realidade social quando esses dados se mostraram relevantes.
} 


\section{Resultados e Discussão}

\section{Associação entre Uso Prejudicial de Substâncias e Comportamento Suicida entre Adolescentes}

Tanto no Brasil quanto em outras regiões do mundo, a ocorrência de comportamentos suicidas entre adolescentes e pessoas jovens tem aumentado consideravelmente (Benincasa \& Rezende, 2006; Borges, Werlang, \& Copatti, 2008; Ores et al., 2012; Van Heeringen, 2001). Benincasa e Rezende (2006) citam que o comportamento suicida comparece como segunda maior causa de internações de indivíduos do sexo feminino na faixa etária entre 10 e 19 anos, assistidos pelo Sistema Único de Saúde (SUS) brasileiro. No que tange à associação entre suicídio e uso abusivo de substâncias, a expressividade do acometimento de adolescentes é transparecida nos dados dos estudos, que compreendem seis dentre os 17 revisados.

Interessados em estudar a correlação entre uso de substâncias e suicídio na adolescência, Pompili et al. (2012) procederam à revisão de literatura nas bases de dados PubMed, Medline, Scopus, PsycLIT e PsycInfo, entre os anos de 1980 e 2011, para atender ao interesse principal de averiguar publicações acerca da epidemiologia e de programas preventivos direcionados a esse público. Como resultado do estudo, feito em Roma, de 17 artigos científicos, os autores apontam que adolescentes com quadro de uso abusivo de drogas que tentaram ou completaram suicídio podem ser caracterizados como pessoas vivenciando eventos de vida estressores, com desordem de humor, problemas interpessoais, suporte social pobre, sentimentos de desesperança e/ou vida solitária. Ademais, os autores sugerem que programas preventivos devam se basear na detecção de fatores de risco associados tanto ao suicídio quanto ao abuso de substâncias, além de buscarem uma combinação de estratégias variadas, como educação individual, programas escolares e tratamentos psicológico e farmacológico.

Dougherty, Hill-Kapturczak, Richard, Mathias e Dawes (2008), em estudo baseado em dados da população norte-americana catalogados por meio de pesquisa nacional sobre uso de drogas, também procederam a revisão de literatura com o objetivo de distinguir trajetórias de desenvolvimento dos comportamentos de uso de substâncias e comportamento suicida, além de descrever fatores de risco e proteção para eles. Os autores mencionam hipóteses que predizem relacionamento bidirecional entre eventos de vida estressantes e desregulagem de marcadores genéticos de serotonina (5-HT), comportamentos de uso de substância e impulsividade e consumo de drogas e tentativas de suicídio. Por fim, ressaltam que a realização de estudos acerca da associação entre uso de substâncias e suicídio deve suscitar melhor entendimento sobre tempo de duração e extensão de fatores de risco e proteção para que, futuramente, outros estudiosos conduzam tratamentos e testem métodos preventivos mais adequados.

De modo diverso ao de Pompili et al. (2012) e Dougherty et al. (2008), os autores americanos Effinger e Stewart (2012) adotaram método empírico para correlacionar suicídio e uso abusivo de álcool e outras drogas entre adolescentes. Utilizaram dados de uma pesquisa norte-americana, a National Survey of Drug Use and Health, para classificar a coocorrência entre sintomas de depressão e abuso de substâncias como preditora de tentativas de suicídio em amostra de 17.705 adolescentes de 12 a 17 anos estratificada por raça, gênero, região e idade.

Para proceder à classificação, Effinger e Stewart (2012) apoiaram-se no Modelo Quadrante de Classificação, composto por seis categorias: sintomas depressivos implícitos, depressão 
clínica, coocorrência de baixa severidade de uso de substâncias e baixa severidade de depressão [Quadrante 1], coocorrência de baixa severidade de uso de substância e alta severidade de depressão [Quadrante 2], coocorrência de alta severidade de uso de substância e baixa severidade de depressão [Quadrante 3], e coocorrência de alta severidade de uso de substâncias e alta severidade de depressão [Quadrante 4]. A partir do manejo das informações, os autores inferiram que adolescentes classificados no Quadrante 4 estavam em maior risco de suicídio que os demais, além dos classificados nos Quadrantes 2 e 3 estarem em riscos idênticos entre si. Com tudo isso, ressaltam a propensão do comportamento suicida entre adolescentes com coocorrência de patologias, até quando os sintomas depressivos são leves. Isso aponta para a necessidade de métodos preventivos primários e secundários e interventivos mais significativos e abrangentes ao nível leve da sintomatologia da depressão, já que serviços específicos para adolescentes com sintomas de depressão em nível leve são geralmente pouco ofertados nos equipamentos públicos concernentes à saúde mental (Effinger \& Stewart, 2012).

De outro modo, Ramchand et al. (2008) examinaram ideação suicida, tentativas de suicídio e subsequentes utilizações de serviços de saúde mental por amostra de 948 adolescentes norte-americanos sob tratamento clínico para abuso de substâncias. Para tanto, procederam a entrevistas no momento da admissão no serviço de saúde e a cada 3 meses ao longo de um ano de tratamento. No que concerne ao pensar em suicídio, verificaram que $30 \%$ dos adolescentes relataram ideação suicida em ao menos uma das cinco entrevistas, enquanto $12 \%$ relataram tentativas de suicídio ao menos uma vez durante o período, o que, em suma, aponta para urgência de aplicação de intervenções precisas entre eles.

Com relação à dita necessidade de propostas interventivas, cabe revisar o estudo de Esposito-Smythers, Spirito, Kahler, Hunt e Monti (2011), que testaram a aplicação de protocolo de tratamento cognitivo-comportamental (I-CBT) em quarenta adolescentes internados em hospitais psiquiátricos e que tinham questões relacionadas ao uso abusivo de substâncias e ao suicídio, bem como em seus familiares, todos selecionados randomicamente. Como resultados, os autores verificaram melhorias em ambas as questões, uso abusivo de álcool e outras drogas e comportamento suicida, mensuráveis a partir de expressivo decréscimo de uso de serviços de saúde adicionais, tais como buscas a atendimentos hospitalares emergenciais.

Diante da visível associação entre uso abusivo de substâncias e suicídio entre adolescentes, parece urgente a necessidade de ações interventivas. Entretanto ela foi verificada em apenas um dos artigos revisados (Esposito-Smythers et al., 2011). Apesar disso, há de ser considerada a relevância dos estudos que se propõem ao conhecimento e à propagação de dados sobre a temática, para que novas ações práticas se voltem efetivamente ao público em questão.

\section{Associação entre Uso Abusivo de Substâncias e Comportamento Suicida em Grupos Socioculturais}

Karch, Barker e Strine (2006) utilizaram dados provenientes do National Violent Death Reporting System (NVDRS), que, em 2004, incluiu dados de 13 estados norte-americanos acerca de indivíduos mortos por causas violentas. Em posse dessas informações, procederam 
ao cálculo da prevalência de abuso de substâncias e doença mental entre vítimas de suicídio pertencentes a diferentes grupos raciais/étnicos. Além disso, buscaram tendências desses grupos em relação à saúde mental e ao abuso de substâncias que poderiam ser utilizadas para melhorias em estratégias de prevenção ao suicídio. Verificaram a ocorrência de 6.865 suicídios cujas raças/etnias eram conhecidas, nos estados e no ano em questão.

Debruçando-se sobre essas ocorrências, os autores supracitados descreveram que, quanto às raças/etnias identificadas entre pessoas que cometeram suicídio, 5.797 (84,4\%) eram não hispânicas brancas; 501 (7,3\%) eram não hispânicas negras; 257 (3,7\%) eram hispânicas e $310(4,5 \%)$ eram pessoas de outros grupos raciais/étnicos. Destaca-se, assim, que o impacto do racismo não pode ser atribuído à maior parte dos casos, já que foram cometidos por pessoas não hispânicas brancas. Esta pesquisa não vê, portanto, de maneira geral, esse impacto no panorama apresentado.

Já no que concerne especificamente ao uso de substâncias na ocasião do cometimento do suicídio, exames toxicológicos apontaram menor teor de álcool entre não hispânicos negros que em qualquer outro grupo, enquanto não hispânicos brancos apresentaram mais consumo de antidepressivos ou opioides e menos de cocaína que os demais. Por fim, quanto aos níveis de utilização de Cannabis e anfetamina, não houve diferenças significativas entre os grupos. Diante desses índices, Karch et al. (2006) concluem ser necessários esforços preventivos ao suicídio, não só para aqueles em abuso de substâncias e com questões de saúde mental, mas também para sujeitos com características individuais, familiares e sociais de grupos raciais/étnicos diferentes.

Kropp et al. (2013) pesquisaram características de 77 participantes autodenominados índios, da planície do norte dos Estados Unidos, que buscaram tratamento para episódio recente de abuso de substâncias em uma clínica urbana. Nesses casos, entre os dados obtidos pela aplicação de instrumento para mensurar a severidade da adiç̧ão entre os participantes, o suicídio compareceu como uma das diversas repercussões negativas do uso de substâncias. Nesse contexto, os autores ressaltam que a idade prematura do uso abusivo foi diretamente correlacionada à vitimização, à deficiência cognitiva e ao comportamento suicida.

Por fim, Taylor, Nguyen, Sinkewicz, Joe e Chatters (2012) se propuseram a examinar a influência do país de origem na comorbidade humor-ansiedade entre caribenhos negros nos Estados Unidos. Para isso, examinaram a correlação entre transtornos psiquiátricos gerais, transtorno por uso de substâncias e comportamento suicida. Tomaram uma subamostra $(n=1.621)$ de caribenhos negros respondentes da National Survey of American Life, procedida pelo Instituto de Pesquisa Social da Universidade de Michigan, em que foram realizadas entrevistas com 6.082 indivíduos de ao menos 18 anos, entre os quais africanos americanos, não hispânicos brancos e negros descendentes de caribenhos eram foco do estudo. Taylor et al. (2012) verificaram que 7,19\% dos 1.621 caribenhos negros respondentes tinham comorbidade entre transtorno de humor e transtorno de ansiedade (8,66\% tinham apenas desordem de humor e $11,46 \%$ apenas de ansiedade). A primeira geração foi menos propensa a apresentar sintomas de transtorno de humor, de ansiedade ou de transtorno por uso de substância ou a se engajar em tentativas de suicídio ao longo da vida, em comparação aos nascidos nos Estados Unidos, país marcado por intensa, mas nem sempre bem-sucedida, luta contra o racismo (Andrews, 1985). No que concerne ao gênero, os autores verificaram 
que homens caribenhos negros foram mais propensos a apresentar transtorno por uso de substância ou apenas transtorno de ansiedade ou tentativas de suicídio do que mulheres dessa nacionalidade. Diante desse quadro, é proposto endereçamento de ações de cuidado em saúde a grupos com maiores riscos de desenvolver desordens, principalmente entre populações subestudadas, como os caribenhos negros.

Em suma, é consenso entre os autores supracitados neste item a necessidade de endereçamento de ações preventivas e de tratamento da comorbidade em questão para subgrupos específicos de maior risco em termos socioculturais, tais como caribenhos negros (Taylor et al., 2012) e índios (Kropp et al., 2013), em vez de minimizar as suas particularidades. Esses textos sugerem endereçamento de ações de modo bem amplo, sem especificar estratégias a serem adotadas.

\section{Correlações entre Outros Transtornos Psiquiátricos, o Uso Abusivo de Substâncias e o Suicídio}

Para além do que concerne a grupos socioculturais particulares, transtornos psiquiátricos como a esquizofrenia não raramente se relacionam tanto ao uso de substâncias (Arias Horcajadas, Sanchez Romero \& PadÝn Calo, 2002) quanto ao suicídio (Chaves, 2000; Louza Neto, \& Moreno, 1993). Kobylecki et al. (2008) afirmam que a associação entre esses fatores pode se relacionar, em parte, a falhas no tratamento com antipsicóticos, invariavelmente indicado para as pessoas com diagnóstico de esquizofrenia. Isso, segundo os autores, acontece porque indivíduos que carregam variantes funcionais dos genes CYP2D6 e CYP2C19 parecem sofrer alterações metabólicas que interferem na absorção de drogas psicoativas, com risco de sua acumulação tóxica ou de sua eliminação demasiadamente rápida.

Nesse contexto, Kobylecki et al. testaram se a comorbidade entre transtorno por uso de substâncias e comportamento suicida seria associada aos genótipos CYP2D6 e CYP2C19 de pessoas com esquizofrenia. Para tanto, 372 indivíduos com esse transtorno foram avaliados a partir de entrevistas clínicas. Como resultado, verificaram não haver associação significativa entre os fatores em teste, o que vem a remontar a necessidade de novas hipóteses para explicar a correlação entre esquizofrenia, transtorno por uso de substâncias e/ou comportamento suicida.

Tolliver e Anton (2015), por sua vez, fornecem uma visão geral sobre avaliações e tratamento de transtornos de humor no contexto do abuso e da dependência de substâncias, sem, porém, detalhar os métodos utilizados para coletar as informações. De todo modo, ressaltam o reconhecimento e manejo de sintomas de desordem de humor em usuários de álcool e/ou outras drogas como desafio para profissionais que lidam com essas pessoas, o que justifica a recomendação de que se realize uma coleta cuidadosa do histórico psiquiátrico desses usuários. Segundo eles, o não reconhecimento de depressão maior ou transtorno bipolar pode aumentar consideravelmente as taxas de recaída no uso e/ou o risco de suicídio.

No contexto supracitado, Tolliver e Anton (2015) ponderam que alguns avanços científicos têm sido alcançados nas últimas décadas, já que pesquisas epidemiológicas têm elucidado a correlação entre transtornos de humor e os transtornos por uso de substâncias. Tal correlação aponta para além das crenças prévias de que os primeiros transtornos seriam meramente resultantes da intoxicação e, portanto, não requereriam tratamento específico. 
Aqui, cabe justificar a inclusão deste artigo entre os revisados: apesar de o suicídio não ter comparecido diretamente relacionado ao transtorno por uso de substâncias, comparece como possível desdobramento dos transtornos de humor, como a depressão, correlacionados pelos autores ao abuso e/ou à dependência. A afirmação desse desdobramento está em concordância com Boschloo et al. (2011), Hess et al. (2012), Kotov et al. (2010), Swendsen et al. (1998) e Vicente et al. (2001).

Na mesma linha de correlação entre depressão, abuso/dependência de drogas e suicídio, Ortíz-Gómez, López-Canul e Arankowsky-Sandoval (2014) objetivaram determinar fatores psicológicos e sociodemográficos associados à depressão e às tentativas de suicídio entre 57 pacientes em tratamento em um centro de atenção mexicano equivalente ao Centro de Atenção Psicossocial Álcool e Drogas (CAPSad) do governo brasileiro. Como instrumentos, utilizaram-se da Mini International Neuropsychiatric Interview (MINI), de questionário de informações gerais, de dados históricos sobre consumo de substâncias, depressão e tentativas de suicídio e de adaptação espanhola da escala de estresse de Holmes e Rathe, para avaliar o impacto de eventos estressores na vida. A partir daí, acessaram, por meio de regressão logística, que $68,4 \%$ dos pacientes apresentavam depressão maior, dos quais $75,4 \%$ já a experienciavam antes do início do abuso de substâncias. Pacientes que tentaram suicídio antes do uso de drogas corresponderam a $26 \%$, enquanto $28,1 \%$ tentaram suicídio dentro do último ano.

Ademais, Ortíz-Gomez et al. (2014) associaram a tentativas atuais de suicídio variáveis anteriores ao uso de drogas: recebimento de diagnóstico de depressão e tentativa anterior de suicídio. Entre tudo isso, é possível presenciar, ainda, um dado bastante relevante: o histórico familiar de abuso de drogas como significativamente relacionado à depressão, o que, segundo os autores, aponta para necessidade de ações preventivas e promocionais no âmbito familiar.

\section{Estudos Interventivos: Estratégias de Prevenção}

Somando-se ao estudo de Esposito-Smythers et al. (2011), outros dois estudos, entre os 18 revisados, remontam à aplicação e/ou à avaliação de propostas interventivas, voltadas à prevenção ao suicídio entre indivíduos com uso abusivo de substâncias.

Conner, Wood, Pisani e Kemp (2013) avaliaram treinamento de prevenção ao suicídio aplicado a profissionais dedicados ao tratamento de pessoas com transtorno por uso de substâncias. Metodologicamente, utilizaram um vídeo de duas horas, baseado em manual prático para o manejo do risco de suicídio, produzido pelo Centro de Tratamento do Abuso de Drogas, da Substance Abuse and Mental Health Service Administration, dos Estados Unidos (Treatment Improvement Protocol, TIP 50; Center for Substance Abuse Treatment, 2009). O protocolo foi aplicado a grupos de 273 profissionais de 18 estados norte-americanos. Os resultados foram avaliados a partir de relatórios produzidos em pré-teste, pós-teste e follow-up após 2 meses. Os autores verificaram mudanças estatisticamente significativas na autoeficácia, conhecimento e frequência de práticas de prevenção ao suicídio, assinalando a relevância do domínio desse tipo de tecnologia por profissionais de serviços e equipamentos de prevenção, atenção e reinserção social de pessoas que fazem uso prejudicial de substâncias. 
Por sua vez, Voss et al. (2013) relatam experiência de prevenção ao suicídio aplicada diretamente a indivíduos com problemas por uso abusivo de álcool e outras drogas. Na ocasião, implementaram um teste-piloto do programa Preventing Addiction Related Suicide a 78 pacientes de serviço norte-americano, para tratamento de transtornos por uso de substâncias. A partir daí, verificaram melhorias significativas em comportamentos de buscar ajuda para si, amigos e familiares com problemas por uso abusivo de substâncias, em comparação ao que ocorria um mês antes da aplicação. Por essa via, ressaltam necessidade da inclusão do tema suicídio no tratamento de adictos a partir de estratégias baseadas em evidências.

\section{Associação entre Uso Abusivo de Substâncias e Suicídio em Cenários Diversos}

Alguns artigos selecionados que não se enquadram primariamente em nenhuma das temáticas expostas anteriormente merecem ser ressaltados em suas especificidades.

Assim, tendo como participantes homens com transtorno por uso de substâncias, Mohammadkhani, Khanipour, Azadmehr, Mobramm e Naseri (2015) avaliaram a probabilidade e os preditores de suicídio. Para tanto, aplicaram instrumentos para mensurar as razões para viver, atenção plena (mindfulness) e escala de probabilidade de suicídio em amostra de 324 indivíduos com as características em xeque, que se encontravam em ambulatório ou prisão iranianos. A partir daí, os dados revelaram prevalência de tentativas de suicídio no cenário ambulatorial de $35 \%$, e de $42 \%$ entre os homens em prisões, o que representa índices significativamente altos. De todo modo, a partir da análise dos instrumentos para mensurar mindfulness e razões para viver, os autores ressaltam que a maneira como algo é pensado (processo) é mais importante do que o que é pensado (conteúdo). Sendo assim, a prevenção ao suicídio entre abusadores ou dependentes de substâncias deve incluir intervenções sobre formas de pensar, tornando-as mais adaptativas e menos geradoras de sofrimento psicológico.

Ries, Yuodelis-Flores, Comtois, Roy-Byrne e Russo (2006), por sua vez, estudaram a admissão de pacientes por comportamento suicida devido à síndrome induzida por substâncias (SIS) em serviço hospitalar psiquiátrico. Nesse contexto, aplicaram formulários estruturados a 5.116 pacientes em internamento entre 1ㅇ de janeiro de 1996 e 30 de junho de 2005 e verificaram que, entre os pacientes com maiores graus de SIS, estavam: homens, desempregados, moradores de rua, não caucasianos e aqueles com diagnósticos de transtornos psiquiátricos outros, fora da ordem da psicose. Nesse sentido, os autores apontam para o endereçamento de ações, pontuando a necessidade de capacitação profissional para prover tratamentos interventivos intensivos para adicção e comportamentos suicidas entre os apontados como pertencentes ao grupo de risco, para além da recorrente tendência - também apontada por Tolliver e Anton (2015) - de profissionais taxarem a associação entre suicídio e adicção como meramente induzida pelas substâncias em uso, sem a urgência de tratamentos específicos.

Já Arendt, Munk-Jørgensen, Sher, Jensen e Signe (2013) estudaram o excesso de mortalidade associado à desordem por uso de Cannabis na Dinamarca, a partir de registros e dos índices padronizados de mortalidade (Standardized Mortality Rates, SMRs) estimados entre diferentes causas de morte. A amostra foi composta por 6.445 pessoas que haviam recebido tratamento para uso abusivo de Cannabis em todas as instituições dinamarquesas 
especializadas, entre 10 de janeiro de 1996 e 31 de dezembro de 2006. Entre os SMRs encontrados, os autores destacam: acidentes; suicídio; homicídio/violência; e causas naturais. Além disso, no que concerne diretamente ao suicídio, os autores verificaram ocorrências cinco vezes mais frequentes durante o acompanhamento da amostra, quando comparada à população em geral. Diante disso, os autores apontam a necessidade de endereçamento de ações interventivas relacionadas ao risco de mortalidade associada ao uso abusivo de Cannabis, que em doses baixas é visto como de pouco a nada ofensivo por alguns autores (e.g., Carlini, 2006).

Por fim, Silveira, Fidalgo, Di Pietro, Santos e Oliveira (2014) relatam o único estudo revisado realizado em cenário brasileiro. O objetivo do estudo foi identificar se o abuso de drogas é fator de risco para escolha de métodos potencialmente mais fatais de tentativas de suicídio, como pular de locais altos, cortar os pulsos ou se enforcar, em oposição a métodos menos perigosos, como ingerir pílulas. Para tanto, procederam a aplicações de entrevista semiestruturada e questionários sociodemográficos com 86 pacientes que sobreviveram a tentativas de suicídio, admitidos na emergência de um hospital geral de São Paulo.

Com os dados obtidos, Silveira et al. (2014) puderam concluir que o uso de drogas foi preditivo para severidade da tentativa de suicídio, como relatado por $81 \%$ dos envolvidos em tentativas mais prejudiciais e por $46,2 \%$ dos que usaram métodos menos prejudiciais. Entre todos esses, entretanto, o uso de drogas no mesmo dia da tentativa de suicídio não foi relacionado à severidade, mas quando o uso se dera no último mês, o que ainda é considerado recente. Com isso, os autores julgam necessário monitorar grupos específicos que apresentem risco de concretizar o suicídio, como os usuários de drogas.

\section{Considerações Finais}

A partir da revisão dos artigos, evidencia-se a associação entre uso abusivo de substâncias e suicídio, o que vem atraindo o interesse de estudiosos. Entretanto, apesar de sua relevância como questão social e de saúde pública, apenas três dos 17 estudos revisados se propõem interventivos. Em contrapartida, estudiosos parecem se preocupar em explorar e sinalizar aspectos urgentes da situação, o que em si compreende um avanço, já que é possível verificar o caráter velado da temática suicídio (Sampaio \& Boemer, 2000) somado ao caráter moralista que, muitas vezes, permeia a temática do uso de drogas (Carlini, 2006) e do suicídio.

Essas propostas de medidas interventivas se apresentam ainda mais escassas sob a perspectiva de ação de promoção à saúde, entendida como dispositivo de transformação social, que concebe que a saúde não depende somente do setor de saúde, mas requer o entrecruzamento dos setores, de saberes e de vivências e envolve o compartilhamento de responsabilidades sociais, incluindo a população geral como participante ativa desse processo. Para a promoção à saúde, portanto, faz-se imprescindível a articulação dos vários setores da sociedade no enfrentamento dos determinantes sociais, políticos, econômicos, educacionais, ambientais, culturais, que estão na gênese do processo saúde-doença (Iglesias, 2009).

Trata-se, como trazido por Guareschi, Lara e Ecker (2016) em estudo sobre encaminhamentos de jovens a tratamentos por uso de drogas, de romper com "padrões de cuidado até então colocados" (p. 34), para iniciar práticas dirigidas à efetivação de direitos constitucionais, 
como acesso à educação e a projetos profissionalizantes, para assegurar que suas vidas sejam protegidas em contexto de retorno a uma situação, muitas vezes, de miséria.

São abordadas nos artigos medidas de prevenção, o que certamente se destacam como de muita importância no cuidado, porém, conforme Iglesias (2009), as ações preventivas e promocionais se complementam, mas não se equivalem, cada qual carrega seu próprio entendimento de saúde, mundo e sujeito. Enquanto a prevenção de doenças, por exemplo, baseia-se no paradigma epidemiológico, o que supõe uma ação antecipada sobre fatores causadores de doença nos indivíduos e nos grupos de pessoas, a promoção à saúde trabalha com um conceito ampliado de saúde, envolvendo os sujeitos na construção de melhores condições de vida (lazer, educação, saneamento básico, alimentação etc.), determinantes à sua saúde.

Ademais, compareceu em alguns estudos revisados (Karch et al., 2006; Silveira et al., 2014; Taylor et al., 2012) o cuidado em assinalar subgrupos de risco para o suicídio entre os usuários de drogas, como: adolescentes; indivíduos pertencentes a grupos socioculturais específicos, tais como os índios e os negros; e indivíduos com diagnósticos de transtornos psiquiátricos, como a esquizofrenia. Ao que tudo indica, então, faz-se mister a implementação de ações endereçadas a essas pessoas, juntamente a novos esforços privados e públicos para o delineamento das urgências que perpassam a comorbidade entre uso abusivo de substâncias/ dependência química e suicídio. Por tudo isso, sugere-se a integração de ações de prevenção de doenças e promoção à saúde para o cuidado com esse problema de saúde pública.

Cabe ainda assinalar as limitações do presente artigo. O número relativamente baixo de estudos revisados prejudicou a possibilidade de resultados mais abrangentes sobre a comorbidade de uso de álcool e outras substâncias e o suicídio. Foram encontrados estudos que relacionam muito a realidade de outros locais no mundo, mas a intenção era de que mais materiais pudessem descrever melhor esse panorama no Brasil. Portanto este estudo sugere que mais pesquisas sejam feitas para entender melhor o fenômeno estudado dentro do Brasil, para assim melhorar o acesso a informações que ajudem pesquisadores e profissionais da área da saúde a criar práticas mais efetivas de prevenção ao abuso de drogas e ao suicídio.

\section{Referências}

Andrews, G. R. (1985). O negro no Brasil e nos Estados Unidos. Lua Nova: Revista de Cultura e Política, 2(1), 52-56. doi: https://doi.org/10.1590/S0102-64451985000200013

Arendt, M., Munk-Jørgensen, P., Sher, L., Jensen, \& Signe, O. W. (2013). Mortality following treatment for cannabis use disorders: Predictors and causes. Journal of Substance Abuse Treatment, 44, 400-406. doi: https://doi.org/10.1016/j.jsat.2012.09.007

Arias Horcajadas, F., Sanchez Romero, S., \& Padýn Calo, J. J. (2002). Influencia del consumo de drogas en las manifestaciones clínicas de la esquizofrenia. Actas Españolas de Psiquiatría, 30(2), 65-73.

Bastos, F. I., Bertoni, N., Hacker, M. A., \& Grupo de Estudos em População, Sexualidade e Aids. (2008). Consumo de álcool e drogas: Principais achados de pesquisa de âmbito nacional, Brasil 2005. Revista de Saúde Pública, 42(1), 109-117. 
Benincasa, M., \& Rezende, M. M. (2006). Tristeza e suicídio entre adolescentes: Fatores de risco e proteção. Boletim de Psicologia, 56(124), 93-110. Disponível em http://pepsic.bvsalud. org/scielo.php?script=sci_arttext\&pid=S0006-59432006000100007\&lng=pt\&tlng=pt

Bessa, M. A., Boarati, M. A, \& Scivoletto, S. (2011). Crianças e adolescentes. In: A. Diehl, D. C. Cordeiro, \& R. Laranjeira (Orgs.), Dependência química: Prevenção, tratamento e políticas públicas (pp. 359-374). Porto Alegre: Artmed.

Borges, V. R., Werlang, B. S. G., \& Copatti, M. (2008). Ideação suicida em adolescentes de 13 a 17 anos. Barbarói, 28, 109. doi: http://dx.doi.org/10.17058/barbaroi.v0i0.1922

Boschloo, L., Vogelzangs, N., Smit, J. H., van den Brink, W., Veltman, D. J., Beekman, A. T., \& Penninx, B. W. (2011). Comorbidity and risk indicators for alcohol use disorders among persons with anxiety and/or depressive disorders: Findings from the Netherlands Study of Depression and Anxiety (NESDA). Journal of affective disorders, 131(1), 233-242. doi: https://doi.org/10.1016/j.jad.2010.12.014

Brasil. (2006). Prevenção do suicídio: Manual dirigido a profissionais das equipes de saúde mental. Disponível em https://bvsms.saude.gov.br/bvs/publicacoes/manual_editoracao.pdf

Brasil. (2017). Padrões de uso de drogas. Eixo Políticas e Fundamentos. Disponível em http:// www.aberta.senad.gov.br/medias/original/201704/20170424-094251-001.pdf

Carlini, E. A. (2006). A história da maconha no Brasil. Jornal Brasileiro de Psiquiatria, 55(4), 314-317. doi: https://doi.org/10.1590/S0047-20852006000400008

Center for Substance Abuse Treatment. (2009). Addressing suicidal thoughts and behaviors in substance abuse treatment. Treatment Improvement Protocol (TIP) Series 50. HHS Publication n. (SMA) 09-4381. Rockville, MD: Substance Abuse and Mental Health Services Administration.

Chaves, A. C. (2000). Diferenças entre os sexos na esquizofrenia. Revista Brasileira de Psiquiatria, 22, 21-22. doi: https://doi.org/10.1590/S1516-44462000000500008

Conner, K. R., Wood, J., Pisani, A. R., \& Kemp, J. (2013). Evaluation of a suicide prevention training curriculum for substance abuse treatment providers based on Treatment Improvement Protocol Number 50 (TIP 50). Journal of Substance Abuse Treatment, 44(1), 13-16. doi: https://doi.org/10.1016/j.jsat.2012.01.008

Déa, H. R. F. D., Santos, E. N., Itakura, E., \& Olic, T. B. (2004). A inserção do psicólogo no trabalho de prevenção ao abuso de álcool e outras drogas. Psicologia: Ciência e Profissão, 24(1), 108-115. doi: https://doi.org/10.1590/S1414-98932004000100012

Dougherty, D., Hill-Kapturczak, N., Richard, D. M., Mathias, C. W., \& Dawes, M. A. (2008). Adolescent suicidal behavior and substance use: Developmental mechanisms. Substance Abuse: Research and Treatment, 2, 13-28. Disponível em https://www.ncbi.nlm.nih.gov/ pmc/articles/PMC2907920/

Effinger, J. M., \& Stewart, D. G. (2012). Classification of co-occurring depression and substance abuse symptoms predicts suicide attempts in adolescents. Suicide \& Life - Threatening Behavior, 42(4), 353-358. doi: https://doi.org/10.1111/j.1943-278X.2012.00092.x

Escorel, S. (1999). Vidas ao léu: Trajetórias de exclusão social. Rio de Janeiro: Editora Fiocruz. Esposito-Smythers, C., Spirito, A., Kahler, C. W., Hunt, J., \& Monti, P. (2011). Treatment of cooccurring substance abuse and suicidality among adolescents: A randomized trial. Journal of Consulting and Clinical Psychology, 79(6), 728-739. 
Guareschi, N. M. F., Lara, L., \& Ecker, D. D. (2016). A internação compulsória como estratégia de governamentalização de adolescentes usuários de drogas. Estudos de Psicologia (Natal), 21(1), 25-35.

Guimarães, J. L., Godinho, P. H., Cruz, R., Kappann, J. I., \& Tosta Jr, L. A. (2004). Consumo de drogas psicoativas por adolescentes escolares de Assis, SP. Revista de Saúde Pública, 38(1), 130-2. doi: https://doi.org/10.1590/S0034-89102004000100018

Hess, A. R. B., Almeida, R. M. M. D., \& Moraes, A. L. (2012). Comorbidades psiquiátricas em dependentes químicos em abstinência em ambiente protegido. Estudos de Psicologia, 17(1), 171-178. doi: https://doi.org/10.1590/S1413-294X2012000100021

Iglesias, A. (2009). Em nome da promoção à saúde: Análise das ações em macrorregião do município de Vitória-ES (Dissertação de Mestrado em Saúde Coletiva, Universidade Federal do Espírito Santo, Vitória, 194p.).

Karch, D. L., Barker, L., \& Strine, T. W. (2006). Race/ethnicity, substance abuse, and mental illness among suicide victims in 13 US states: 2004 data from the National Violent Death Reporting System. Injury Prevention, 12(suppl 2), ii22-ii27. Disponível em https://www. ncbi.nlm.nih.gov/pubmed/17170166

Kobylecki, C. J., Hansen, T., Timm, S., Wang, A., Jakobsen, K. D., Sørensen, H. J., . . . Werge, T. (2008). The impact of CYP2D6 and CYP2C19 polymorphisms on suicidal behavior and substance abuse disorder among patients with schizophrenia: A retrospective study. Therapeutic Drug Monitoring, 30(3), 265-270.

Kotov, R., Gamez, W., Schmidt, F., \& Watson, D. (2010). Linking "big" personality traits to anxiety, depressive, and substance use disorders: A meta-analysis. Psychological Bulletin, 136(5), 768. doi: https://doi.org/10.1037/a0020327

Kropp, F., Somoza, E., Lilleskov, M., Moccasin, M., Moore, M., Lewis, D., . . Winhusen, T. (2013). Characteristics of northern plains American Indians seeking substance abuse treatment in an urban, non-tribal clinic: A descriptive study. Community Mental Health Journal, 49(6), 714-721. doi: https://doi.org/10.1007/s10597-012-9537-7

Louza, M. R., Neto, \& Moreno, R. A. (1993). Diagnóstico diferencial e tratamento farmacológico de depressão em esquizofrenia. Jornal Brasileiro de Psiquiatria, 42(supl. 1), 47-51s. Disponível em https://pesquisa.bvsalud.org/portal/resource/pt/psi-10265

Mohammadkhani, P., Khanipour, H., Azadmehr, H., Mobramm, A., \& Naseri, E. (2015). Trait mindfulness, reasons for living and general symptom severity as predictors of suicide probability in males with substance abuse or dependence. Iranian JournalofPsychiatry, 10(1), 56. Disponível em https://www.ncbi.nlm.nih.gov/pmc/articles/PMC4434430/

Ores, L. D. C., Quevedo, L. D. A., Jansen, K., Carvalho, A. B. D., Cardoso, T. A., Souza, L. D. D. M., ... Silva, R. A. D. (2012). Risco de suicídio e comportamentos de risco à saúde em jovens de 18 a 24 anos: Um estudo descritivo. Cadernos de Saúde Pública, 28(2), 305-312. doi: https://doi.org/10.1590/S0102-311X2012000200009

Organização Mundial de Saúde. (2000). Prevenção do suicídio: Manual para médicos clínicos gerais. Genebra: Organização Mundial de Saúde.

Ortíz-Gómez, L. D., López-Canul, B., \& Arankowsky-Sandoval, G. (2014). Factors associated with depression and suicide attempts in patients undergoing rehabilitation for substance abuse. Journal of Affective Disorders, 169, 10-14. doi: https://doi.org/10.1016/j.jad.2014.07.033 
Pompili, M., Serafini, G., Innamorati, M., Biondi, M., Siracusano, A., Di Giannantonio, M., . . Möller-Leimkühler, A. (2012). Substance abuse and suicide risk among adolescents. European Archives of Psychiatry and Clinical Neuroscience, 262(6), 469-485. Disponível em https://www.ncbi.nlm.nih.gov/pubmed/22290639

Ramchand, R., Griffin, B. A., Harris, K. M., Mccaffrey, D. F., Morral, A. R. S., \& Howard, J. (2008). A prospective investigation of suicide ideation, attempts, and use of mental health service among adolescents in substance abuse treatment. Psychology of Addictive Behaviors, 22(4), 524-532. doi: https://doi.org/10.1037/a0012969

Reed, K. P., Nugent, W., \& Cooper, R. L. (2015). Testing a path model of relationships between gender, age, and bullying victimization and violent behavior, substance abuse, depression, suicidal ideation, and suicide attempts in adolescents. Children and Youth Services Review, 55, 128-137. doi: https://doi.org/10.1016/j.childyouth.2015.05.016

Ries, R. K., Yuodelis-Flores, C., Comtois, K. A., Roy-Byrne, P. P., \& Russo, J. E. (2008). Substance-induced suicidal admissions to an acute psychiatric service: Characteristics and outcomes. Journal of Substance Abuse Treatment, 34(1), 72-79.

Rother, E. T. (2007). Revisão sistemática X revisão narrativa. Acta Paulista de Enfermagem, 20(2), v-vi. doi: https://doi.org/10.1590/S0103-21002007000200001

Saide, O. L. (2011). Depressão e uso de drogas. Revista Hospital Universitário Pedro Ernesto, 10(2). Disponível em http://www.e-publicacoes.uerj.br/index.php/revistahupe/article/ view/8852

Sampaio, M. A., \& Boemer, M. R. (2000). Suicídio: um ensaio em busca de um des-velamento do tema. Revista da Escola de Enfermagem da USP, 34(4), 325-331. doi: https://doi. org/10.1590/s0080-62342000000400002

Silva, L. H. P. D., Borba, L. D. O., Paes, M. R., Guimarães, A. N., Mantovani, M. D. F., \& Maftum, M. A. (2010). Perfil dos dependentes químicos atendidos em uma unidade de reabilitação de um hospital psiquiátrico. Escola Anna Nery Revista de Enfermagem, 14(3), 585-90. doi: https://doi.org/10.1590/S1414-81452010000300021

Silveira, D. X., Fidalgo, T. M., Di Pietro, M., Santos, J. G., \& Oliveira, L. Q. (2014). Is drug use related to the choice of potentially more harmful methods in suicide attempts? Substance Abuse: Research and Treatment, 8, 41-43.

Swendsen, J. D., Merikangas, K. R., Canino, G. J., Kessler, R. C., Rubio-Stipec, M., \& Angst, J. (1998). The comorbidity of alcoholism with anxiety and depressive disorders in four geographic communities. Comprehensive Psychiatry, 39(4), 176-184. Disponível em https://www.ncbi.nlm.nih.gov/pubmed/9675501

Taylor, R. J., Nguyen, A., Sinkewicz, M., Joe, S., \& Chatters, L. (2012). Comorbid mood and anxiety disorders, suicidal behavior, and substance abuse among black caribbeans in the U.S.A. Journal of African American Studies, 17(4), 409-425.

Tolliver, B. K., \& Anton, R. F. (2015). Assessment and treatment of mood disorders in the context of substance abuse. Dialogues in Clinical Neuroscience, 17(2), 181-90. Disponível em https://www.ncbi.nlm.nih.gov/pubmed/26246792

VanHeeringen, C.(2001). Suicideinadolescents.InternationalClinicalPsychopharmacology,16, S1-S6. Disponível em http://journals.Iww.com/intclinpsychopharm/Abstract/2001/03002/ Suicide_in_adolescents.2.aspx 
Vicente, S., Nunes, A., Viñas, C., Freitas, D., \& Saraiva, C. B. (2001). Depressão, ideação suicida e desesperança em doentes alcoólicos. Psiquiatria Clínica, 22(1), 85-93. Disponível em http://hdl.handle.net/10400.4/876

Voss, W. D., Kaufman, E., O'Connor, S. S., Comtois, K. A., Connor, K. R., \& Ries, R. K. (2013). Preventing addiction related suicide: A pilot study. Journal of Substance Abuse Treatment, 44(5), 565-569. doi: https://doi.org/10.1016/j.jsat.2012.10.006

Recebido em: 17/01/2018

Última revisão: 17/05/2018

Aceite final: $23 / 10 / 2018$

\section{Sobre os autores:}

Juliana Gomes da Cunha Baltar - Doutoranda do Programa de Pós-Graduação em Psicologia da Universidade Federal do Espírito Santo. E-mail: juliana88gomes@gmail.com, Orcid: http://orcid.org/0000-0003-1412-4904

Alexandra Iglesias- Professora do Departamento de Psicologia da Universidade Federal do Espírito Santo. E-mail: leiglesias@gmail.com, Orcid: http://orcid.org/0000-0001-7188-9650

Elizeu Batista Borloti- Professor doutor do Departamento de Psicologia Social e do Desenvolvimento e do Programa de Pós-Graduação em Psicologia da Universidade Federal do Espírito Santo. E-mail: borloti@hotmail.com, Orcid: http://orcid.org/0000-0002-6217-6541 